\title{
Gonarthrose
}

\section{Ist die arthroskopische Therapie sinnlos?}

Die arthroskopische Lavage und das intraartikuläre Debridement sind eine verbreitete Maßnahme bei Gonarthrosebeschwerden. Langfristig bringe dies im Vergleich zur konservativen Therapie nichts, hat nun eine hochrangig publizierte Studie* ergeben. Ist damit die Arthroskopie generell infrage gestellt?, fragten wir Dr. Alwin Jäger, Orthopäde an der Berufsgenossenschaftlichen Unfallklinik in Frankfurt am Main.

MMW: Kirkley et al.* stellten fest, dass die Arthroskopie bei Gonarthrosepatienten keinen Zusatznutzen im Vergleich zu einer optimalen konservativen Therapie hat. Überrascht Sie das?

Jäger: Nein, wenn ich mir das Patientenkollektiv der Studie ansehe: Im Durchschnitt waren die Teilnehmer etwa $60 \mathrm{Jah}$ re alt, der Body-Mass-Index war mit 30 bis $32 \mathrm{~kg} / \mathrm{m}^{2}$ deutlich erhöht. Diese Patienten sind nach Arthroskopie ein bis drei Monate zufrieden, weil z. B. Abriebteilchen, die die Entzündung unterhalten, herausgespült und dadurch die Beschwerden gelindert werden. Aber natürlich treten relativ rasch die gleichen Beschwerden wieder auf. Übergewicht und chronische Kniebinnenschäden bleiben ja. Sie werden auch mit der konservativen Therapie nicht behoben, mit dem Unterschied, dass diese fortgesetzt werden kann. Solche Patienten benötigen ein multimodales Therapiekonzept.

MMW: Wird mit der Studie die Arthroskopie als diagnostisches und therapeutisches Instrument infrage gestellt? Jäger: Auf keinen Fall! So ist das aber in der Öffentlichkeit kommuniziert worden. Printmedien haben berichtet: „Arthroskopie nützt nichts" oder „Arthroskopie meist überflüssig". In der Tat wird die Arthroskopie bei älteren Patienten zu oft eingesetzt. Bei ihnen hat sie sicher nicht den Stellenwert wie bei Patienten jungen und mittleren Alters. Leider ist das sehr undifferenziert dargestellt worden.

*Kirkley A et al.: A Randomized Trial of Arthroscopic surgery for Osteoarthritis of the Knee. N Engl J Med 2008;359:1097-1107
MMW: Für welche Patienten ist denn die arthroskopische Behandlung angezeigt? Jäger: Das sind vor allem Patienten mittleren Alters mit Knorpelschäden. Neben den bildgebenden Verfahren ist die Arthroskopie dort sehr wichtig, um eine Diagnostik des Knorpelschadens vornehmen zu können. Wo genau liegt der Knorpelschaden? Wie ist er entstanden - auf dem Boden eines Meniskusschadens, ist es ein Unfall gewesen oder eine degenerative Veränderung? Für diese Patienten ist die Arthroskopie ein Segen. Denn unmittelbar im Anschluss an die Diagnose kann man z.B. den Meniskusschaden und anschließend den Knorpelschaden beheben. Eine rein diagnostische Arthroskopie sollte eigentlich nicht mehr durchgeführt werden, da die präoperativen diagnostischen Möglichkeiten in den letzten Jahren verbessert worden sind.

V Vor allem Patienten mittleren Alters mit Knorpelschäden profitieren von der Arthroskopie.

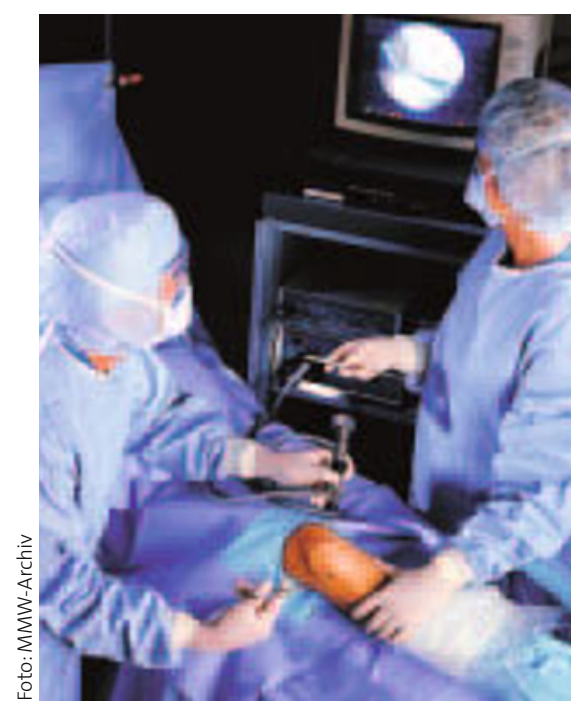

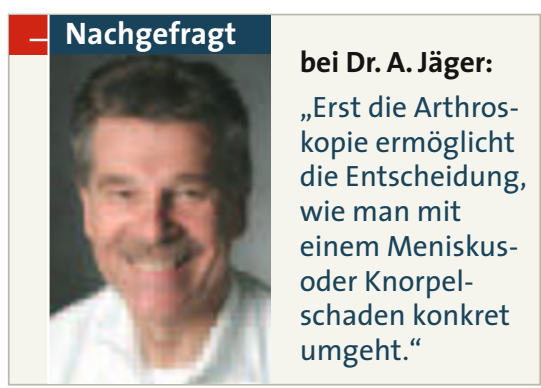

MMW: Was sagen Sie der 48-jährigen Patientin mit Kniebeschwerden nach Verdrehtrauma, die in der Zeitung gelesen hat: „Gelenkspiegelung bringt nichts“, den Eingriff ablehnt und lieber eine Kernspintomografie haben möchte?

Jäger: Natürlich nutzen wir im Einzelfall verschiedene diagnostische Instrumente, angefangen von der genauen Anamnese und klinischen Untersuchung sowie dem konventionellen Röntgenbild. Erst dann beginnen wir zu überlegen: Welche weiteren Informationen brauchen wir? Selbstverständlich ist die Kernspintomografie ein wichtiges Instrument, etwa für die Operationsplanung. Wenn ich mich für eine Gelenkspiegelung entscheide, sollte sie in aller Regel auch die Therapie beinhalten. Aber erst die Arthroskopie ermöglicht mir die Entscheidung, wie ich mit einem eingeklemmten oder angerissenen Meniskus, einem verletzten Kreuzband oder einem Knorpelschaden konkret umgehe.

MMW: Wie lautet also Ihr Fazit zu der Studie „Arthroskopie versus konservative Therapie bei Gonarthrose“?

Jäger: Es handelte sich um ein sehr spezielles und eingeschränktes Patientenkollektiv. Ausgeschlossen waren Patienten mit Meniskusschäden, mit entzündlichen und postentzündlichen Arthritiden, Varus- oder Valgusdeformitäten, vorangegangenen Knietraumata und anderem. Die Ergebnisse können und sollten also nicht generalisiert werden. Gegebenenfalls kann man selbst alte Patienten erfolgreich arthroskopisch operieren, sodass sie sogar wieder altersgerecht Sport ausüben können.

- Interview: Dr. Thomas Meißner

Haben Sie noch Fragen an Dr. Jäger? Sie erreichen ihn über die MMW-Redaktion: Fax: 089-4372-1399

E-Mail:moreano@urban-vogel.de 